\title{
POSTER
}

\section{Pigmentation palatine associée à un traitement par Imatinib : à propos d'un cas}

\section{Fourcade $C^{1}$, Dauzat $A^{2}$, Le Guennec $B^{2}$, Cloitre $A^{2}$, Kimakhe $S^{3}$, Rouaud $\mathrm{M}^{4}$, Lesclous $\mathrm{Ph}^{5}$, Boedec $A^{2}$}

1 - DESCO

2 - AHU

3 - MCU-PH

4 - Etudiante

5 - PU-PH, ; Unité Fonctionnelle de Chirurgie Orale, Service d'Odontologie Restauratrice et Chirurgicale, CHU de Nantes, Faculté de Chirurgie Dentaire de Nantes (France)

Introduction

L'Imatinib (Glivec ${ }^{\circledR}$ ) est un inhibiteur de la tyrosine kinase indiqué dans le traitement des patients atteints de leucémie myéloïde chronique en particulier après échec de l'interféron alpha. Différentes manifestations endobuccales induites par les thérapies ciblées anticancéreuses sont répertoriées. La littérature scientifique ne rapporte qu'une quinzaine de cas d'hyperpigmentation palatine de la muqueuse buccale associés à un traitement par Imatinib. Ces cas d'hyperpigmentation endobuccale sont moins fréquents que les troubles d'hypopigmentation ou de dépigmentation exobuccales. Si le diagnostic clinique, appuyé des antécédents médicaux de ces patients, ne laisse que peu de doute, l'histologie et la physiopathologie de ce processus sont moins fréquemment décrites.

\section{Observation}

Le cas d'une femme de 82 ans, suivie pour une réhabilitation globale de la cavité buccale au centre de soins dentaires du CHU de Nantes, traitée par Imatinib (400 mg par jour) depuis 12 ans pour une leucémie myéloïde (LM) en phase chronique est décrit (traitement antérieur par interféron alpha puis par hydroxycarbamide). Elle est également traitée par darbepoéitine alplha, un antianémique et par acide acétylsalycilique suite à un double pontage et par bisoprolol, un bêtabloquant, pour une hypertension artérielle. Elle est non fumeuse. La patiente présente une lésion palatine asymptomatique sous forme d'une pigmentation mélanique diffuse de l'ensemble de la muqueuse recouvrant le palais dur, de couleur brune. De découverte fortuite, il est donc difficile de déterminer son ancienneté. Aucune autre localisation n'est décelée lors de l'examen clinique. Une biopsie de cette lésion a été réalisée afin d'en étudier les caractéristiques histologiques. L'examen histologique montre en coloration HES un épithélium malpighien kératinisé sans prolifération mélanocytaire ce qui est confirmé par l'immunomarquage avec l'anticorps antiprotéine S100. Le chorion sous-jacent renferme des pigments noirs positifs à la coloration de PERLS. Toute prolifération maligne est écartée. Une simple surveillance a été instaurée. Cette lésion ne montre aucun potentiel évolutif après 6 mois. Le traitement par imatinib a été maintenu. 


\section{Discussion}

Plusieurs publications récentes font état d'hyperpigmentations muqueuses orales liées à l'imatinib, la première décrite en 2009. Sur la quinzaine de cas répertoriés, toutes sont situées exclusivement au niveau de la muqueuse recouvrant le palais dur. L'imatinib en se fixant sur le récepteur c-Kit jouerait un rôle dans la migration, la prolifération et la différenciation mélanocytaire. C'est ce qu'illustre le cas clinique présenté.

\section{Conclusion}

Aucune évolution maligne de ce type de lésion n'ayant été décrite, une simple surveillance clinique doit être mise en place.

\section{Lectures utiles}

Lewis DM. Diffuse pigmentation of the palate. J Okla Dent Assoc 2009. 100 :24-5.

Mattson U. et coll. Oral pigmentation in the hard palate associated with imatinib mesylate therapy : a report of three cases. Oral Surg Oral Med Oral Pathol Oral Radiol Endod 2011. $111: 12-6$.

Campana F et Ordioni U. Pigmentation palatine associée à un traitement par imatinib. Med Bucc Chir Bucc 2014. 20 :275-7. 